\title{
Die SE im Steuerrecht
}

Professor Dr. Wolfgang Schön und

Dr. Clemens Philipp Schindler, LL. M.

I. Einführung

1. Rechtsentwicklung . . . . . . . . . 1

a) Gesetzgebungsarbeiten der EG/

EWG ............. 1

aa) Allgemeines ......... 1

bb) Keine Steuerregeln in der SEVO ........... 4

cc) Steuerliche Richtlinien ... . . 7

dd) Gemeinsame körperschaftsteuerliche Bemessungsgrundlage . 10

b) Gesetzgebungsarbeiten in der Bundesrepublik Deutschland . . . . . 14

2. Die SE im Recht der Europäischen Gemeinschaft . . . . . . . . . 17

a) Grundfreiheiten . . . . . . . . . 17

b) Steuerliche Richtlinien . . . . . . . 20

c) Verhältnis Grundfreiheiten - Richtlinien .............. 25

d) Die SE und EFTA/EWR . . . . . . . . 35

II. Die SE im nationalen Steuerrecht . . . 38

1. Direkte und indirekte Steuern . . . . . 38

2. Subjektive Steuerpflicht . . . . . . . 43

a) Unbeschränkte Steuerpflicht . . . 43

aa) Die SE als Kapitalgesellschaft i.S.v. § 1 Abs. 1 Nr. 1 KStG . . . 43

bb) Geschäftsleitung oder Sitz im Inland

cc) Auseinanderfallen von Sitz und Geschäftsleitung ........ 53

dd) Ausländische SE mit Geschäftsleitung im Inland . . . . 56

ee) Beginn und Ende der Steuerpflicht ........... 59

b) Beschränkte Steuerpflicht . . . . . . 61

c) Steuerbefreiungen . . . . . . . . 65

3. Objektive Steuerpflicht . . . . . . 67

a) Einkommen, Einkommensermittlung und Einkommensverwendung 67 aa) Qualifikation der Einkünfte . . 67

(1) Unbeschränkt steuerpflichtige SE . . . . . . . . . 67

(2) Beschränkt steuerpflichtige SE ............

bb) Maßgeblichkeit der Handelsbilanz für die steuerliche Gewinnermittlung ....... 71

cc) Körperschaftsteuerliche Gewinnmodifikationen . . . . . . 75 (1) Allgemeines . . . . . . 75
(2) § 10 Nr. 4 KStG (Abzugsfähigkeit von Aufsichtsratsvergütungen u. Ä.) . . . . . 76

(3) Offene und verdeckte Gewinnausschüttungen . . . . 79

b) Organschaft . . . . . . . . . . 81

aa) SE mit Sitz und Geschäftsleitung im Inland . . . . . . . . . 81

bb) SE mit Sitz im Ausland und Geschäftsleitung im Inland . . 84

(1) Organträger . . . . . . . . . 84

(2) Organgesellschaft . . . . . 85

cc) SE mit Sitz im Inland und Ort der Geschäftsleitung im Ausland ............ 88

(1) Organträger . . . . . . . . 88

(2) Organgesellschaft . . . . . 89

dd) SE ohne Sitz oder Ort der Geschäftsleitung im Inland . . . . 91

(1) Organträger ......... 91

(2) Organgesellschaft . . . . . 94

III. Die SE im Doppelbesteuerungsrecht . 97

IV. Die Sitzverlegung der SE . . . . . . . 100

1. Wegzug einer SE . . . . . . . . . 100

a) Besteuerung der Gesellschaft . . . . 100

aa) Anwendbare Rechtsvorschrif-

ten . . . . . . . . . . 100

(1) $\S 12 \mathrm{KStG} \ldots \ldots . . . \ldots 102$

(2) EG-Grundfreiheiten . . . . . 106

(3) Fusionsbesteuerungs-RL . . 112

bb) Der Wechsel von der unbeschränkten in die beschränkte Steuerpflicht . . . . . . 115

(1) Der Wechsel in die beschränkte Steuerpflicht im Rahmen von $\S 12$ Abs. 1 KStG . . . . . . . . . 115

(2) Gemeinschaftsrechtliche Aussagen zum Wechsel in die beschränkte Steuerpflicht ......... 116

(3) Verlegung des Satzungssitzes ........... 118

(4) Verlegung des Verwaltungssitzes . . . . . . . . . 123

(5) Verlegung des Ortes der Geschäftsleitung . . . . . . . 125

(6) Konsequenzen für die Anwendung von $\S 12$ Abs. 1 KStG . . . . . . . . 126 
cc) Die Belegenheit von Vermögen im Rahmen von $\S 12$ Abs. 1

KStG . . . . . . . . . . . . 129

(1) Inländisches Vermögen . . 130

(2) Verlagertes Vermögen . . . 133

(a) Wechsel in die ausländische Steuerhoheit . . . 133

(b) Fortbestehendes Besteuerungsrecht für die "stillen Reserven"? . . . . . . 138

(3) Ausländisches Vermögen . . 143

dd) Die Aufdeckung stiller Reserven . . . . . . . . . . 146

ee) Keine Anwendbarkeit des Besteuerungsaufschubs nach $\S 4 \mathrm{~g}$ EStG . . . . . . . . . . 149

ff) Vereinbarkeit der "Sofortbesteuerung" mit Gemeinschaftsrecht? . . . . . . . . 150

gg) Verlustvorträge . . . . . . 158

b) Besteuerung der Anteilseigner . . . 163

2. Zuzug einer SE . . . . . . . . . . 173

a) Besteuerung der Gesellschaft . . . 174

aa) Wechsel beschränkte/unbeschränkte Steuerpflicht . . . . 174

bb) Zugangsbewertung von Betriebsvermögen . . . . . . 176

cc) Verlustvorträge . . . . . . . 179

b) Besteuerung der Anteilseigner . . . 182

3. Sitzverlegung im EU-Ausland mit Inlandsbezug . . . . . . . . . . . . 184

4. Sitzverlegung in EWR-Staaten . . . . 186

5. Sitzverlegung in Drittstaaten . . . . . 188

V. Die Gründung einer SE . . . . . . . . 191

1. Die Gründung einer SE durch Ver-

schmelzung . . . . . . . . . . . 192

a) Die Hinausverschmelzung . . . . . 194

aa) Gesetzliche Grundlagen . . . . 195

(1) Deutsches Steuerrecht . . 196

(2) Europäisches Sekundärrecht ......... 203

(3) Europäisches Primärrecht . 208

bb) Beteiligte Gesellschaften . . . . 210

(1) Die relevanten Normen . . 210

(2) Wesentliche Fallgruppen . . 215

cc) Rechtsfolgen bei der übertragenden Gesellschaft . . . . . . 219

(1) Der Regelfall: Übertragende und übernehmende Gesellschaft sind in der EU ansässig . . . . . . . . . . 219

(2) Ansatz des gemeinen Wertes in der steuerlichen Schlussbilanz . . . . . . . . . . 223

(3) Voraussetzungen für den Ansatz der Buchwerte . . . 225

(4) Antrag der übertragenden Gesellschaft . . . . . . . 229

(5) Beschränkung des deutschen Besteuerungsrechts
(6) Vereinbarkeit der Sofortbesteuerung mit den Grundfreiheiten? . . . . . . . . 239

(7) Bilanzielle Auswirkungen . 242

dd) Rechtsfolgen bei der übernehmenden Gesellschaft . . . . . . 245

(1) Übernahme der Bilanzwerte der übertragenden Gesellschaft . . . . . . . . 245

(2) Ausschluss des Verlustvortrages . . . . . . . . . 247

(3) Behandlung von Anteilen der übernehmenden an der übertragenden Gesellschaft 249

(4) Übernahmefolgegewinn . . . 253

ee) Rechtsfolgen bei den Anteilseignern . . . . . . . . . . . . 254

ff) Die übernehmende Gesellschaft ist in einem dem EWR zugehörenden EFTA-Staat ansässig . . . . . . . . . . 260

gg) Die übernehmende Gesellschaft hat ihre Geschäftsleitung in einem Drittstaat (außerhalb von EU/EWR) . . . . . . 265

b) Die Hereinverschmelzung . . . . . . 269

aa) Verschmelzung zur Aufnahme und zur Neugründung . . . . . 269

bb) Steuerfolgen bei der übertragenden Gesellschaft . . . . . . . . . 272

cc) Steuerfolgen bei der übernehmenden Gesellschaft . . . . . . 276

dd) Steuerfolgen bei den Anteilseignern . . . . . . . . . . . 281

c) Die Auslandsverschmelzung mit Inlandsbezug . . . . . . . . . . . 282

aa) Inländisches (Betriebs-|Vermögen der übertragenden Ge-

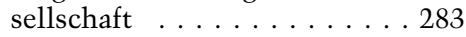

bb) Behandlung der Anteilseigner . 286

2. Die Gründung einer Holding-SE . . . . 287

a) Gesetzliche Grundlage . . . . . . . 287

b) Begünstigte Rechtsformen nach der Fusionsbesteuerungs-RL und dem SEStEG . . . . . . . . . . . 291

c) Ansässigkeitsvoraussetzungen nach der Fusionsbesteuerungs-RL und dem SEStEG . . . . . . . . . 296 aa) Fusionsbesteuerungs-RL . . . . 296 bb) UmwStG . . . . . . . . . . . . 297

(1) Übernehmender Rechtsträger . . . . . . . . . 298

(2) Erworbener Rechtsträger . . 301

(3) Einbringende Personen . . . 303

d) Erwerb einer Stimmrechtsmehrheit an Anteilen einer Kapitalgesellschaft . . . . . . . . . . . . . . 306 aa) Fusionsbesteuerungs-RL . . . 307 bb) Deutsches Umwandlungssteuerrecht . . . . . . . . . 312

e) Anteilsgewährung der übernehmenden Gesellschaft . . . . . . . 318 
f) Buchwertfortführung . . . . . . . 321

aa) Wertansatz bei der Gesellschaft . . . . . . . . . 321

bb) Wertansatz bei den Gesellschaftern ........... 322

(1) Wahlrecht zur Fortführung der Buchwerte . . . . . . 322

(2) Zwingende Aufdeckung stiller Reserven . . . . . . . 329

(3) Gegenausnahmen ... . . . 331

g) Keine Rückwirkungsfiktion . . . . 336

h) Nachträgliche Besteuerung . . . . 337

i) Zusammenfassende Darstellung . 338

aa) Ebene der Holding-SE . . . . . . 339

bb) Ebene der Gründungsgesellschaften . . . . . . . . . 344

cc) Ebene der Gesellschafter . . . . 347

dd) Ergebnis . . . . . . . . . . 351

3. Die Gründung einer gemeinsamen

Tochtergesellschaft . . . . . . . . 352

a) Gründungsformen . . . . . . . . . 352

aa) Beteiligte Rechtsträger . . . . 352

bb) Bargründung . . . . . . . . . . 354

cc) Sachgründung . . . . . . . 356

b) Persönlicher Geltungsbereich der

Fusionsbesteuerungs-RL und des

UmwStG

361 c) Ansässigkeit der beteiligten

Rechtsträger . . . . . . . . . . 363

aa) Einbringender . . . . . . . . 365

bb) Übernehmender Rechtsträger . 372

d) Gegenstand der Einbringung . . . 374

e) Anteilsgewährung der übernehmenden Gesellschaft . . . . . . . . 380

f) Buchwertfortführung . . . . . . . 381

aa) Bewertung des eingebrachten Betriebsvermögens . . . . . . 382

bb) Bewertung der gewährten An-

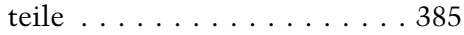

g) Nachträgliche Besteuerung . . . . 387

aa) Veräußerung durch den Einbringenden . . . . . . . 388

bb) Veräußerung durch die übernehmende Gesellschaft . . . . . 394

cc) Siebenjahresfrist . . . . . . . . 397

dd) Alte "einbringungsgeborene" Anteile . . . . . . . . . . . . . 398

h) Ausländische Betriebsstätte . . . . . 399

i) Rückwirkungsfiktion . . . . . . . 400

j) Zusammenfassende Darstellung . . 403

aa) Ebene der SE . . . . . . . . . 405

bb) Ebene der Gründungsgesellschaften . . . . . . . . . . 4 409

4. Die Gründung einer SE durch Formwechsel . . . . . . . . . 415

Literatur: $A ß$ mann, Steuerrechtliche Aspekte der Gründung und Sitzverlegung einer Europäischen Gesellschaft (Societas Europaea), 2006 (zit.: Steuerrechtliche Aspekte); Benecke, Internationalisierung des Ertragssteuerrechts durch das SEStEG - ein Überblick, StuB 2007, 3; Benecke/ Schnitger, Letzte Änderungen der Neuregelungen des UmwStG und der Entstrickungsnormen durch das SEStEG, IStR 2007, 22; Benecke/Schnitger, Neuregelung des UmwStG und der Entstrickungsnormen durch das SEStEG, IStR 2006, 765; Benz/Rosenberg, Einbringungsvorgänge nach dem Regierungsentwurf des SEStEG, BB-Spezial 8/2006, 51; Bilitewski, Gesetz über steuerliche Begleitmaßnahmen zur Einführung der Europäischen Gesellschaft und zur Änderung weiterer steuerrechtlicher Vorschriften (SEStEG), FR 2007, 57; Blumenberg/Schäfer (Hrsg.), Das SEStEG, 2007; Blumers/Kinzl, Änderungen der Fusionsrichtlinie: Warten auf den EuGH, DB 2005, 971; Cordewener, Das Abkommen über den europäischen Wirtschaftsraum: Eine unerkannte Baustelle des deutschen Steuerrechts, FR 2005, 236; Debatin, Die Steuerharmonisierung in der EWG in Form der Konzern-Besteuerungs-Richtlinie, DStZ/A 1969, 146; Diller/Grottke, Die Konzeption von Teilwert und gemeinem Wert - dargestellt am Beispiel des Wechsels vom Teilwert zum gemeinen Wert im Rahmen des SEStEG, StuSt 2007, 69; Dörfler/Rautenstrauch/Adrian, Verlustnutzung bei Verschmelzung von Körperschaften vor und nach Änderung des $§ 12$ Abs. 3 UmwStG durch das SEStEG, BB 2006, 1657; Dörfler/Rautenstrauch/Adrian, Einbringungen in eine Kapitalgesellschaft nach dem SEStEG-Entwurf, BB 2006, 1711; Dötsch/Pung, SEStEG: Die Änderung des UmwStG, DB 2006, 2704 (Teil I), 2763 (Teil II); Dötsch/Pung, SEStEG: Die Änderungen des KStG, DB 2006, 2648; Eckl, Wechsel von beschränkter und unbeschränkter Steuerpflicht bei Kapitalgesellschaften, 2006; M. Fischer in MünchKomm. AktG, 2. Aufl. 2006, Die Besteuerung der Europäischen Aktiengesellschaft; Förster/Lange, Grenzüberschreitende Sitzverlegung der Europäischen Aktiengesellschaft aus ertragsteuerlicher Sicht, RIW 2002, 585; Förster/Lange, Steuerliche Aspekte der Gründung einer Europäischen Aktiengesellschaft (SE), DB 2002, 288; Frotscher, Zur Vereinbarkeit der „Betriebsstättenbedingung“ bei Sitzverlegung und grenzüberschreitender Umwandlung mit den Grundfreiheiten, IStR 2006, 65; Gammi, EU Taxation and the Societas Europaea - Harmless Creature or Trojan Horse?, ET 2004, 35; Goergen, Unternehmensbesteuerung aus europäischer Sicht, JbFfSt 1985/1986, 59; Hageböke/Käbisch, Zur Ausdehnung des Anwendungsbereichs des UmwStG i.d.F. SEStEG-E auf Grund der Diskriminierungsverbote in Art. 24 OECD-MA, IStR 2006, 849; Hagemann/Burkhard/Ropohl/Viebrock, Das neue Konzept der Verstrickung und Entstrickung sowie die Neufassung des Umwandlungssteuergesetzes, NWB 2007, 